\title{
ANÁLISIS ACÚSTICO DE LA LONGITUD VOCÁLICA EN EL INGLÉS CRIOLLO DE LIMÓN
}

\author{
Mario Portilla
}

\begin{abstract}
RESUMEN
Este articulo constituye el primer análisis fonético acústico del inglés criollo de Limón (Costa Rica). El análisis acústico de diversos espectrogramas ha permitido establecer con certeza que la longitud vocálica constituye un rasgo fonético distintivo en esta lengua.

Palabras clave: Lenguas criollas, criollos ingleses, inglés criollo de Limón, fonética acústica, longitud vocálica.
\end{abstract}

\begin{abstract}
In this article the first acoustic analysis on Limonese Creole English (Costa Rica) has been carried out. The acoustic analysis of several spectrograms shows unequivocally that vowel length is a phonetic distinctive trait in this language.

Key words: Creole languages, English-based Creoles, Limon Creole English, acoustic phonetics, vowel length.
\end{abstract}

\section{Antecedentes}

Hasta el presente, todas las descripciones de las vocales del inglés criollo de Limón han sido de índole articulatoria. Terry Wolfe (1970) y Fernando Wright $(1974,1975)$ presentan un inventario de las vocales y de las consonantes de esta lengua, sin llevar a cabo una descripción fonética detallada de los segmentos. Más bien, solo se limitan a transcribir los sonidos aludidos según los símbolos de alfabetos fonéticos convencionales.

Dr. Mario Portilla. Profesor Catedrático. Escuela de Filología, Lingüística y Literatura. Universidad de Costa Rica.

Correo electrónico: marioportilla2000@yahoo.com

Recepción: 22- 5- 2011

Aceptación: 26- 6- 2011 
Terry Wolfe (1970) presenta una lista de nueve segmentos vocálicos simples (cuadro 1), distinguidos por los rasgos $[ \pm$ alto, \pm bajo, \pm tenso, \pm anterior y \pm posterior $]$ y dos diptongos $/$ ai, oi $/{ }^{1}$.

\section{Cuadro 1}

Sistema vocálico según Wolfe (1970)

$\begin{array}{rccc} & \text { [+anterior] } & \text { [-ant., }- \text { post.] } & \text { [+posterior] } \\ \text { [+alto] [+tenso] } & \mathrm{i} & & \mathrm{u} \\ {[\text {-tenso] }} & \mathrm{I} & & \mathrm{U} \\ \text { [-alto, [+tenso] } & \mathrm{e} & \partial & \mathrm{o} \\ \text { [-bajo] [-tenso] } & \varepsilon & \mathrm{a} & \\ \text { [+bajo] } & & \mathrm{a}\end{array}$

Fernando Wright $(1974,1975)$ tampoco realiza un análisis fonológico propiamente dicho. Solamente propone valores fonético-fonológicos ("la simbolización fonética tradicional") para ocho segmentos vocálicos (cuadro 2), que pueden ser distinguidos por los rasgos [ \pm alto, \pm bajo, \pm tenso, \pm anterior], y cuatro diptongos / ai, ie, əu, uə /.

\section{Cuadro 2}

Sistema vocálico según Wright $(1974,1975)$

$\begin{array}{lcc} & \text { [+anterior }] & \text { [-anterior }] \\ \text { [+alto] [+tenso] } & \mathrm{i} & \mathrm{u} \\ \quad \text { [-tenso }] & \mathrm{I} & \mathrm{v} \\ \text { [-alto, }- \text { bajo } & \mathrm{e} & \mathrm{\partial} \\ \text { [+bajo] } & \mathrm{a} & \mathrm{D}\end{array}$

Anita Herzfeld $(1978,2002)$ y Mario Portilla $(1993,1995,1996)$ realizan análisis más detallados de la fonología del inglés criollo de Limón.

Herzfeld $(1978,2002)$ postula la existencia de cinco vocales simples (cuadro 3), que contrastan entre sí por los rasgos distintivos [ \pm alto, \pm bajo, \pm anterior], cuatro diptongos (también llamados por ella transiciones ascendentes y descendentes) / ij, aj, uw, ow /, dos transiciones complejas / ijh, wowh /, y una vocal doble / aa /. ${ }^{2}$

\section{Cuadro 3}

Sistema vocálico según Herzfeld $(1978,2002)$

\begin{tabular}{|c|c|c|}
\hline & [+anterior] & [-anterior] \\
\hline [+alto] [+tenso] & $\mathrm{i}$ & $\mathrm{u}$ \\
\hline [-alto] [-bajo] & e & o \\
\hline [+bajo] & & \\
\hline
\end{tabular}

En cuanto a las vocales / i, u, e /, Herzfeld señala que son "similares" a las que ocurren en inglés estándar en las palabras bit, book y bet respectivamente.

Igualmente, menciona equivalencias de ciertos diptongos o transiciones entre el inglés criollo de Limón y el inglés estándar, como en el caso de / ij /, el cual describe de esta manera: "Diptongo que comienza con una vocal no redondeada alta anterior y está seguida por una transición aún más alta y anterior. Es el sonido del IE / biyt / 'beet' 'remolacha' ” (Herzfeld 2002: 139). 
Portilla (1993) presenta una oposición entre cinco vocales cortas y cinco vocales largas, que se distinguen por los rasgos [ \pm alto, \pm bajo, \pm anterior] (cuadro 4). Además, señala la existencia de dos diptongos descendentes / aI, $\Lambda \mho / .^{3}$

\section{Cuadro 4}

Sistema vocálico según Portilla $(1993,1996)$

\begin{tabular}{|c|c|c|c|c|}
\hline \multirow[b]{2}{*}{ [+alto $][+$ tenso $]$} & \multicolumn{2}{|c|}{$\begin{array}{c}{[+ \text { +anterior }]} \\
{[- \text { largo } /[+ \text { largo }]}\end{array}$} & \multicolumn{2}{|c|}{$\begin{array}{c}{[- \text { anterior }]} \\
{[- \text {-argo }] /[+ \text { largo }]}\end{array}$} \\
\hline & I & i: & $v$ & $\mathrm{u}$ \\
\hline [-alto] [-bajo] & $\mathrm{e}$ & Ie & $\Lambda$ & vo \\
\hline [+bajo] & & a: & $\mathrm{a}$ & \\
\hline
\end{tabular}

En su descripción, Portilla (1993: 90) señala que "las vocales cortas se manifiestan, generalmente, como vocales cualitativamente flojas [-tenso]”. Sin embargo, indica también que son frecuentes las variantes fonéticas tensas, sobre todo cuando estas se encuentran en medio de dos consonantes sordas, como en / fut / $\rightarrow$ [ fu?t ] pie. ${ }^{4}$

Por otro lado, en cuanto a las vocales largas indica que estas se hallan compuestas de dos moras. Cada mora equivale a una vocal simple. Así, aunque las vocales largas de altura máxima / is, u: / y los diptongos ascendentes / ie, vo / suelen ser cualitativamente tensos, "frecuentemente la primera mora de la vocal larga [y el primer elemento del diptongo ascendente] puede manifestarse como fonéticamente floja, especialmente tras consonantes no obstruyentes" (Portilla 1995: 91), como en / ridd / $\rightarrow$ [ riid ] leer; / ruid / $\rightarrow$ [ roud ] raíz; / liek / $\rightarrow$ [ liek ] lago; / nuo / $\rightarrow$ [ nuo ] saber.

Dado que, realmente en estos casos, es solo por el valor fonético de la segunda mora que una vocal larga de altura máxima se distingue de un diptongo descendente, este autor interpreta que los diptongos ascendentes / ie, vo / son los correlatos fonéticos de altura media de vocales largas.

En síntesis, aunque las interpretaciones fonológicas del sistema vocálico del inglés criollo de Limón de todos los autores son distintas, las descripciones tanto de Herzfeld como de Portilla reconocen diferencias fonemáticas debidas a la longitud de los segmentos, lo cual no ocurre en las transcripciones llevadas a cabo por Wolfe y Wright. ${ }^{5}$

La diferencia fundamental entre los análisis de Herzfeld y de Portilla estriba en que la primera autora describe fonéticamente la longitud vocálica como vocales tensas seguidas o precedidas de consonantes aproximantes (o semiconsonantes) (v.gr. / ij, uw / $\rightarrow$ [ ij, uw ] ), mientras que Portilla considera que la longitud vocálica se expresa por medio de moras, cuya cualidad fonética puede variar (v.gr. / i, u: / $\rightarrow$ [ ii $\sim$ Ii, uu $\sim$ vu ] ). Ambos autores coinciden en que las vocales largas de altura baja conservan constante la misma cualidad vocálica: / aa / $\rightarrow$ [ aa ] (Herzfeld); / a: / $\rightarrow$ [ ee ] (Portilla).

A continuación, se presenta una lista de las equivalencias de los segmentos vocálicos según la simbología utilizada por los cuatro autores mencionados.

$\begin{array}{ccccl}\text { Wolfe } & \text { Wright } & \text { Herzfeld } & \text { Portilla } & \\ \text { I } & \text { I } & \mathrm{i} & \mathrm{I} & \text { 'bit' pedazo } \\ \mathrm{U} & \mathrm{U} & \mathrm{u} & \mathrm{U} & \text { 'book' libro } \\ \varepsilon & \mathrm{e} & \mathrm{e} & \mathrm{e} & \text { 'bet' apostar }\end{array}$




\begin{tabular}{|c|c|c|c|c|c|}
\hline ə & ə & o & $\Lambda$ & 'cut' & cortar \\
\hline $\mathrm{a}$ & $\mathrm{a}$ & $\mathrm{a}$ & $\mathrm{a}$ & 'back' & espalda \\
\hline $\mathrm{i}$ & $\mathrm{i}$ & $\mathrm{ij}$ & i: & 'beat' & golpear \\
\hline $\mathrm{u}$ & $\mathrm{u}$ & uw & u: & 'root' & raíz. \\
\hline e & ie & ijh & Ie & 'name' & nombre \\
\hline $\mathrm{o}$ & uə & wowh & vo & 'know' & saber \\
\hline ๑ & $\mathrm{D}$ & aa & $a:$ & 'aal' & todo \\
\hline aI/ oI & ai & aj & aI & 'like' & gustar \\
\hline- & əu & ow & $\Lambda U$ & 'cow' & vaca ${ }^{6}$ \\
\hline
\end{tabular}

\section{Propósito de la investigación}

El propósito fundamental de esta investigación es analizar espectrogramas de una muestra de habla del inglés criollo de Limón con el fin de determinar empíricamente la existencia o no de longitud vocálica en esta lengua. Además, mediante este análisis, se intenta precisar cuáles son los correlatos fonéticos de esta posible longitud vocálica.

La muestra consiste en una lista de vocablos y de frases que fueron educidos a una informante en agosto de 2009.7 La sesión fue llevada a cabo en el laboratorio de fonética del Departamento de Lingüística de la Universidad de Costa Rica, bajo la supervisión de la Dra. Sandra Schwab de la Universidad de Ginebra. ${ }^{8}$ La muestra fue grabada digitalmente y convertida en archivos .wav. Estos archivos fueron analizados espectrográficamente utilizando el programa de análisis acústico PRAAT (versión 5.2.25) (www.praat.org).

\section{La longitud vocálica}

\subsection{Las vocales bajas}

Desde el punto de vista fonológico, las vocales de altura baja se oponen por cantidad, como se nota en las siguientes parejas mínimas:

$\begin{array}{ll}\text { / bak / 'back' espalda : } & \text { / ba:k / 'bark' ladrar } \\ \text { / pat / 'pot' olla : } & \text { / pa:t / 'part' parte } \\ \text { / dag / 'dog' perseguir : } & \text { / da:g / 'dog' perro } \\ \text { / sa / 'sir' señor : } & \text { / sa: / 'saw' sierra } \\ \text { / at / 'hot' caliente : } & \text { / ait / 'heart' corazón }\end{array}$

Los espectrogramas muestran que las vocales bajas largas duran en promedio el doble de las correspondientes vocales cortas cuando aparecen en palabras pronunciadas en aislamiento. Este hecho se nota claramente al comparar los espectrogramas 1 y 2 , referidas a las palabras / bak / 'back' espalda con una vocal corta y / ba:k / 'bark' ladrar con una vocal larga.

En estos ejemplos, la vocal corta / a / dura 0,140 milisegundos, mientras que la vocal larga / a: / tarda 0,295 milisegundos. En este caso, la vocal larga se extiende aproximadamente doble del tiempo que la vocal corta. 
Espectrograma 1

La vocal / a / en palabra / bak / 'back' espalda

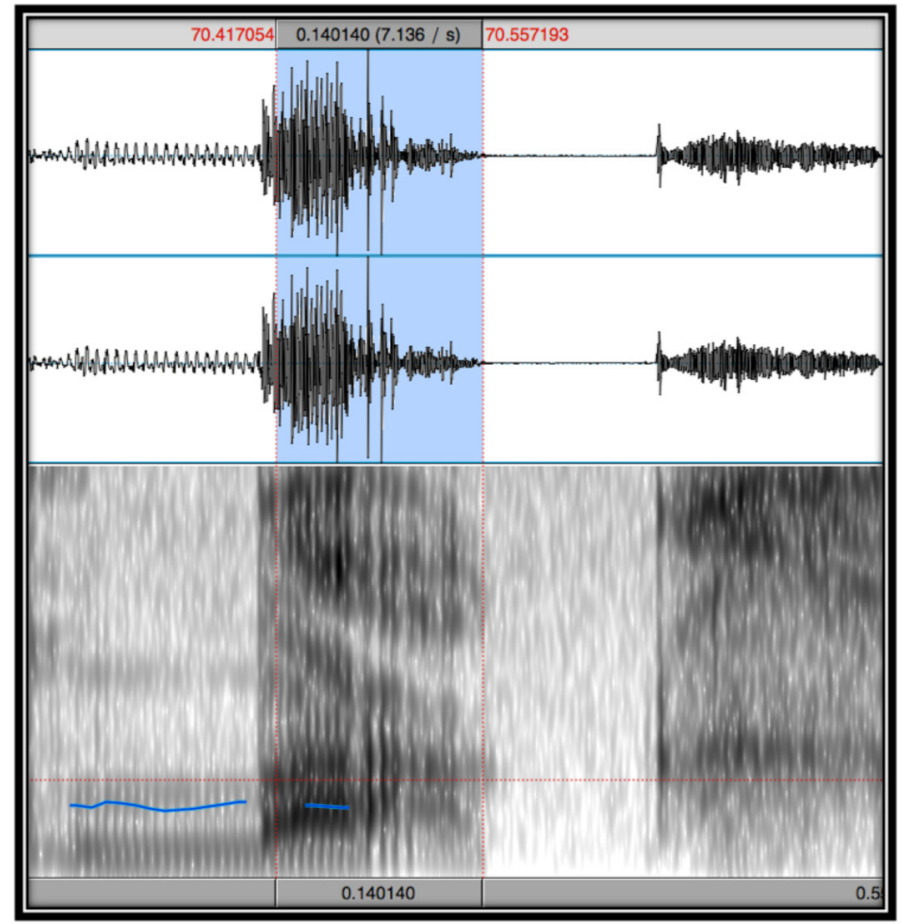

Espectrograma 2

La vocal / a: / en palabra / ba:k / 'bark' ladrar

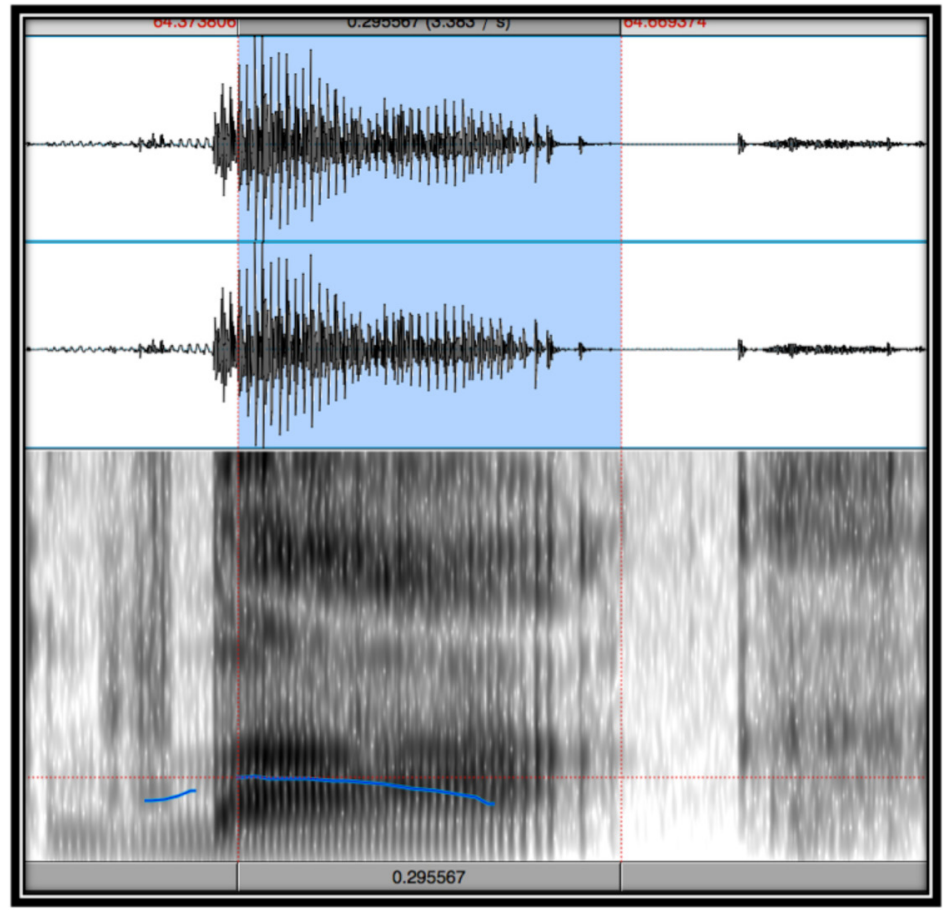


De manera interesante, sin embargo, la diferencia en la duración total de la palabra entre ambos rubros no es tan grande. La palabra / bak / 'back' espalda dura 0,554 milisegundos, mientras que la palabra / ba:k / 'bark' ladrar lo hace por 0,738 milisegundos. Es decir, la palabra con vocal larga dura solo aproximadamente una tercera parte más que la palabra con vocal corta.

También, el espectrograma 2 muestra que la vocal larga está compuesta de dos partes o moras. Esto se nota claramente en el oscilograma arriba del espectrograma, que presenta longitudes de onda mayores en la primera mora que en la segunda. El espectrograma 2 revela, además, que la vocal conserva prácticamente igual sus formantes durante toda su producción. Esto significa que ambas moras presentan una misma cualidad vocálica.

El primer formante (F1) muestra claramente, en ambos espectrogramas, que estas dos palabras contienen vocales bajas. El F1 alcanza una frecuencia de $976 \mathrm{~Hz}$ (herzios) en la palabra espalda y de $975 \mathrm{~Hz}$ en la palabra ladrar. Estos valores son los más bajos registrados para vocales en esta lengua.

El segundo formante (F2) indica que estas vocales bajas son bastante centrales. El F2 llega a una frecuencia de $1814 \mathrm{~Hz}$ en la palabra espalda y de $1888 \mathrm{~Hz}$ en la palabra ladrar. En esta lengua, estos promedios se encuentran exactamente en el medio de los extremos anterior y posterior registrados para la mayoría de otras vocales.

Por último, es interesante señalar que ambos espectrogramas exhiben una oclusión glotal después de las vocales y antes de las consonantes oclusivas. Este fenómeno de intrusión de una consonante oclusiva glotal entre vocales y consonantes oclusivas sordas fue formulado como una regla fonológica por primera vez por Portilla (1993: 91). De esta forma, la transcripción fonética correspondiente de estas palabras queda como sigue:

$$
\begin{aligned}
& \text { / bak / } \rightarrow \text { [ be?k ] espalda } \\
& \text { / baik / } \rightarrow \text { [ ber?k ] espalda }
\end{aligned}
$$

\subsection{La vocales altas}

Las vocales de altura máxima se oponen fonológicamente entre sí tanto por la posición de la lengua como por la longitud de los segmentos.

Los siguientes pares mínimos muestran diferencias de sentido por longitud y por cambios en posición de la lengua:

$\begin{array}{ll}\text { / bit / 'bit' pedazo : } & \text { / bi: / 'beat' golpear } \\ \text { / si / 'see' ver : } & \text { / si: / 'sea' mar } \\ \text { / tu / 'too' también : } & \text { / tui: / 'two' dos } \\ \text { / pit / 'pit' hoyo : } & \text { / put / 'put' poner } \\ \text { / si: / 'sea' mar: } & \text { / su: / 'sue' demandar }\end{array}$

Los espectrogramas 3 y 4 muestran que, en el caso de las vocales anteriores, la diferencia de cantidad entre las vocales cortas y largas es, igualmente, de más del doble. En estos ejemplos, la vocal corta / I / en la palabra / bit / dura 0,107 milisegundos, mientras que la vocal larga / i: / en la palabra / bit / tarda 0,219. Sin embargo, como se ha mencionado antes para las vocales bajas, la diferencia en la duración entre ambas palabras es apenas de una tercera parte. La palabra / bit / dura 0,502 milisegundos y la palabra / bi.t / tarda 0,742. 
Espectrograma 3

La vocal / I / en palabra / bit / 'bit' pedazo

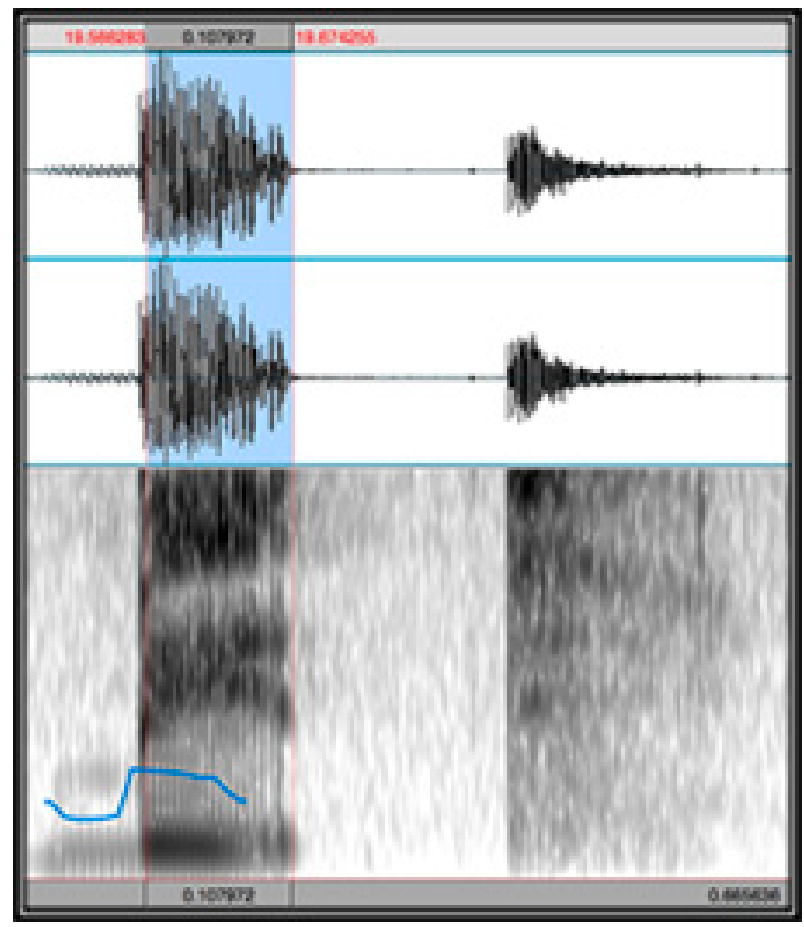

Espectrograma 4

La vocal / i: / en palabra / birt / 'beat' golpear

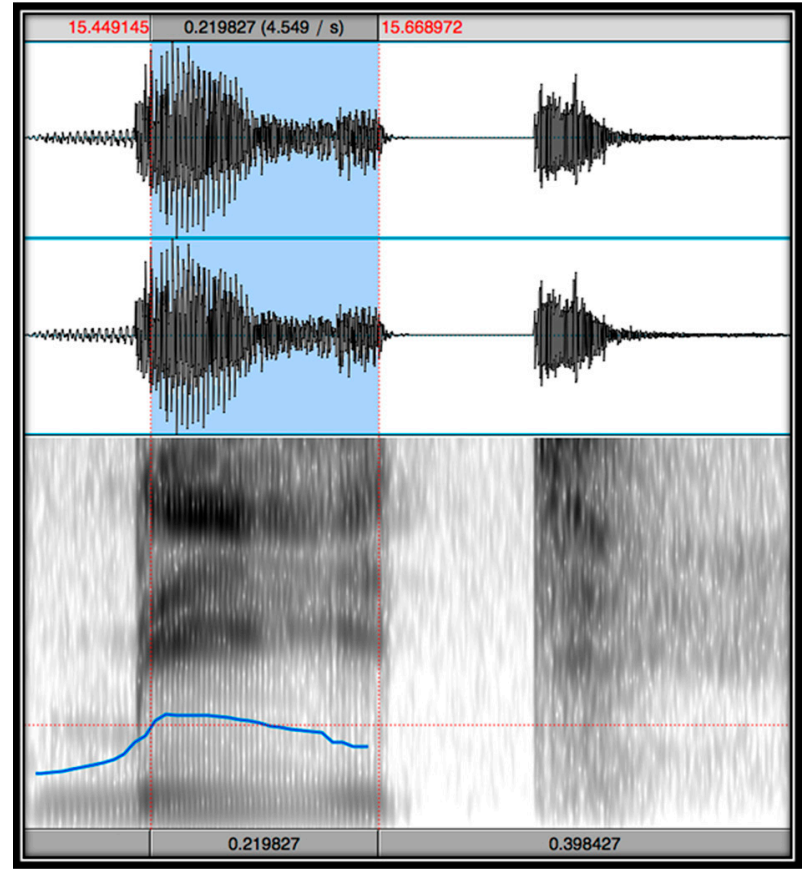


En cuanto a la realización fonética de la vocal larga, el espectrograma 4 revela claramente que está dividida en dos moras. La primera mora alcanza prácticamente la primera mitad de la vocal (un primer lapso de 0,105 milisegundos) y la segunda mora ocupa la segunda mitad (un segundo lapso de 0,114 milisegundos). Esta división aparece, sin dudas, mostrada en el oscilograma, que presenta longitudes de onda mayores en el primer lapso que en el segundo. Además, el espectrograma muestra una mayor energía en el primer lapso que en el segundo.

Por otra parte, es importante señalar que las dos moras presentan una cualidad vocálica un tanto diferente. Tal como lo describiera articulatoriamente Portilla (1993: 91), la primera mora parece consistir de una vocal alta, anterior, un poco más floja [ i ] y la segunda mora de una vocal alta, anterior, más tensa [ i ].

Esto no solo es posible comprobarlo auditivamente al separar las dos moras, sino que aparece mostrado por el comportamiento del primero y segundo formantes (F1 y F2) del espectrograma 5. La frecuencia promedio de F1 de la vocal larga es de $346 \mathrm{~Hz}$ (herzios). Sin embargo, la frecuencia máxima de la F1 de la vocal de la primera mora es de $398 \mathrm{~Hz}$ y la de la segunda mora es de $346 \mathrm{~Hz}$. Por otro lado, la frecuencia de F2 alcanza un promedio de 2483 $\mathrm{Hz}$ en toda la vocal larga. Sin embargo, la frecuencia máxima de F2 de la vocal en la primera mora es de $2533 \mathrm{~Hz}$, mientras que la vocal en la segunda mora es de $2623 \mathrm{~Hz}$. Esto quiere decir que la vocal de la primera mora es un poco más abierta (correspondiente a una frecuencia más alta de F1) y menos anteriorizada (correspondiente a una frecuencia más baja de F2) que la vocal de la segunda mora. Esto significa que la vocal de la primera mora es un tanto más floja que la vocal de la segunda.

Espectrograma 5

Formantes de la vocal / is / en palabra / bitt / 'beat' golpear

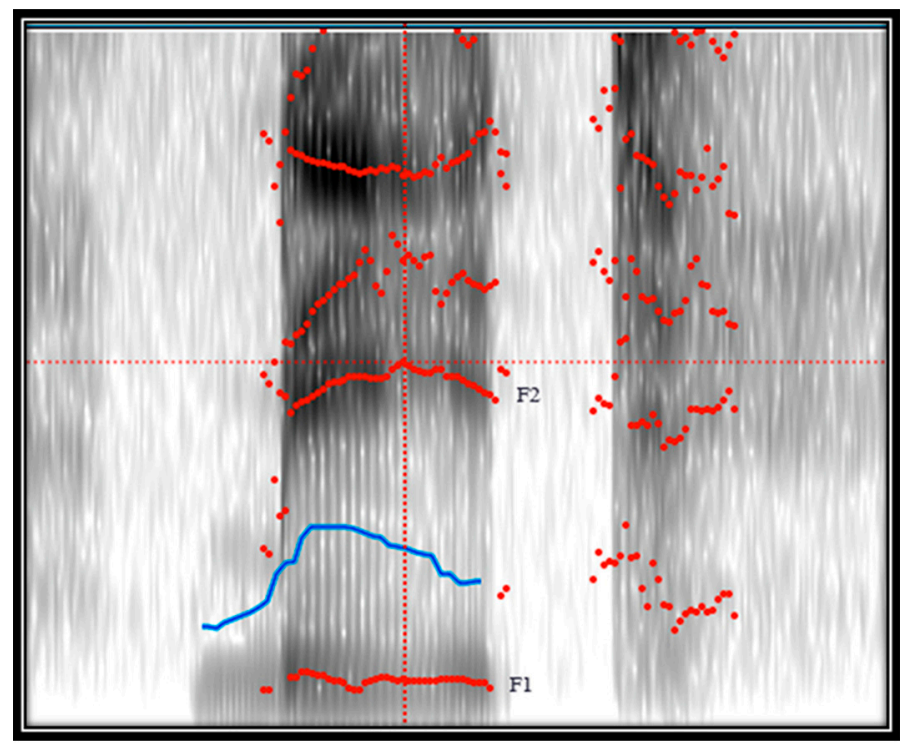

Por otra parte, el espectrograma 6, con la pronunciación de la palabra / si: / 'sea' mar, muestra que, en este contexto, la vocal larga conserva más estable la cualidad de las dos moras [ sii ]. 


\section{Espectrograma 6}

Formantes de la vocal / i: / en palabra / si: / 'sea' mar

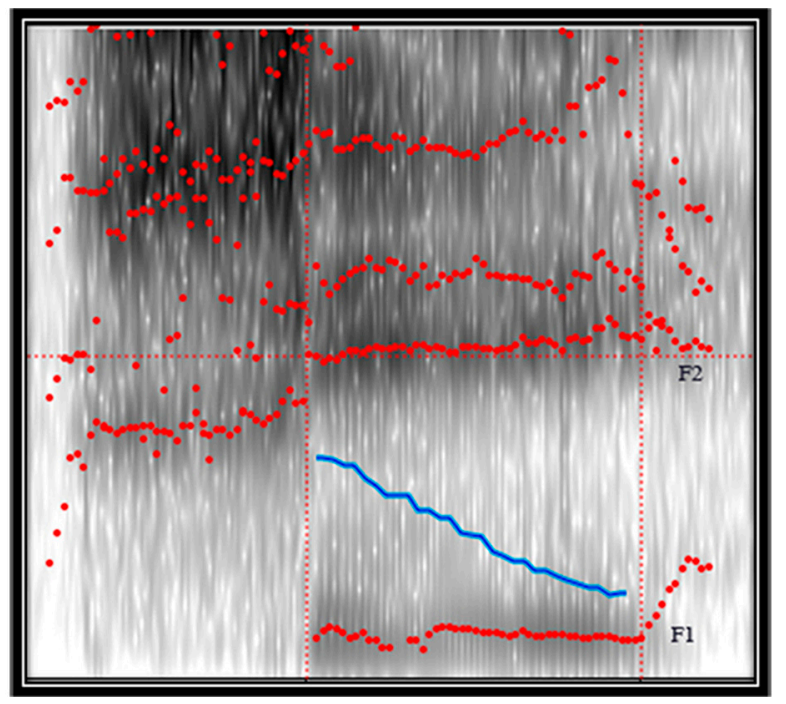

Además, es importante mencionar que en los espectrogramas 5 y 6 no aparecen indicios de las transiciones aproximantes o semiconsonánticas aducidas por Herzfeld (2002) (vid. supra la lista de equivalencias de los segmentos vocálicos).

Respecto de las vocales posteriores, los espectrogramas 7 y 8 muestran las diferencias en cantidad entre las vocales posteriores [ $v$ ] y [ u: ] en las palabras / tu / 'too' también y / tu: / 'two' dos.

\section{Espectrograma 7}

La vocal / $v$ / en palabra / to / 'too' también

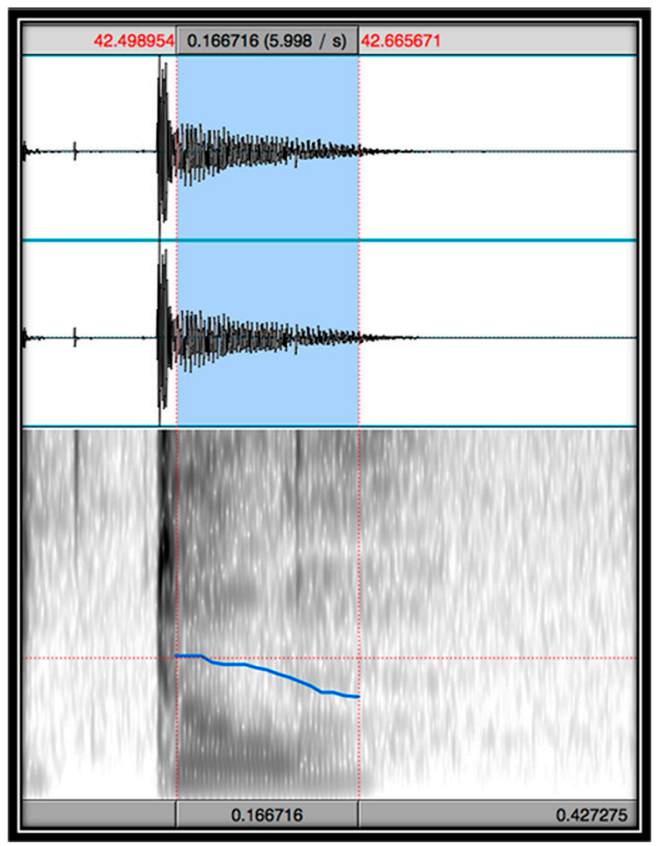


Espectrograma 8

La vocal / u: / en palabra / tu: / 'two' dos

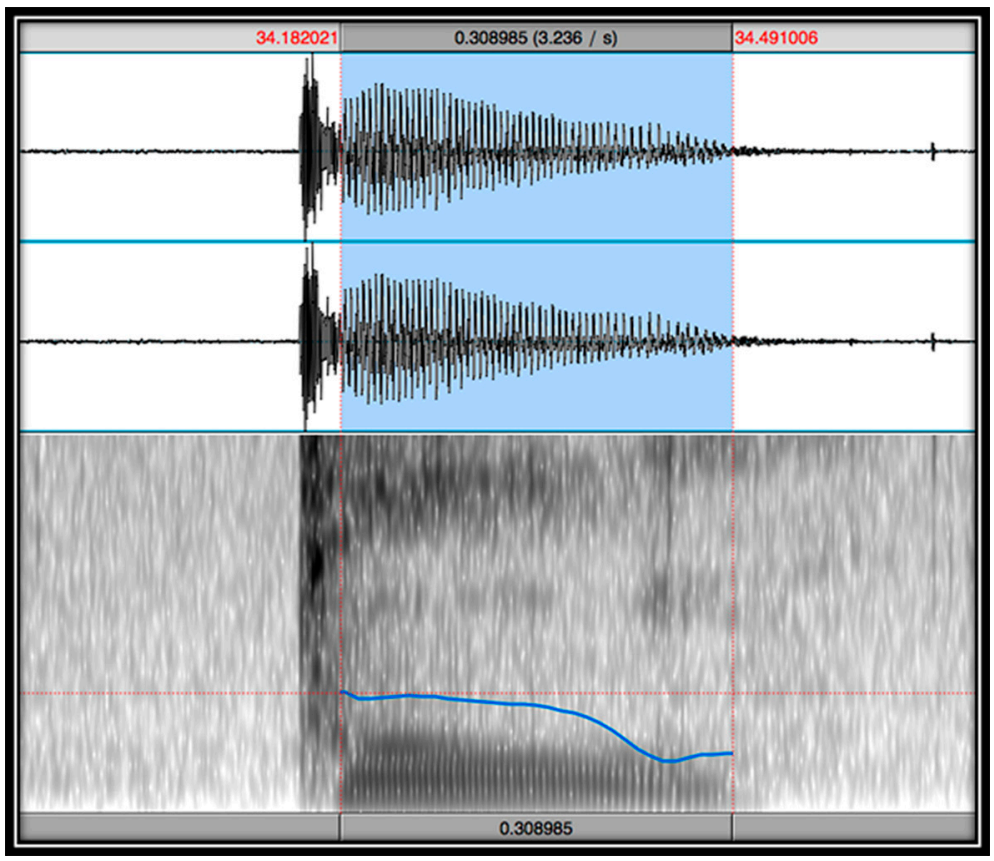

La palabra / tu / 'too' también dura 0,166 milisegundos y la palabra / tu: / 'two' dos tarda 0,308 milisegundos. La diferencia en longitud entre ambas palabras es, como en otros casos, de prácticamente el doble de cantidad.

Las frecuencias del F1 para la vocal / v / es de $655 \mathrm{~Hz}$ y para la vocal / u: / es de $510 \mathrm{~Hz}$. Esto indica que se producen a una altura baja en la cavidad oral, especialmente la vocal corta. Las frecuencias del F2 para la vocal / $v$ / es de $1504 \mathrm{~Hz}$ y para la vocal / u: / es de $1882 \mathrm{~Hz}$.

El espectrograma 9 tampoco presenta indicios de la aparición de transiciones aproximantes o semiconsonánticas, sino más bien una cualidad vocálica estable en toda la vocal larga.

\subsection{La vocales medias}

Las vocales de altura intermedia se oponen fonológicamente entre sí tanto por la posición de la lengua como por la longitud de los segmentos.

Los siguientes pares mínimos muestran diferencias de significado por longitud y por cambios en posición de la lengua:

\section{/ bed / 'bed' cama: \\ / bıd / 'bird' pájaro: ${ }^{9}$ \\ / bed / 'bed' cama : \\ / bied / 'beard' barba}

\author{
/ bied / 'beard' barba \\ / buod / 'board' tabla \\ / bsd / 'bird' pájaro \\ / buod / 'board' tabla
}

La diferencia de cantidad entre las vocales cortas y largas (expresadas como un diptongos descendentes) es de un poco menos del doble en el caso de las vocales medias. En cuanto a las vocales medias posteriores, los espectrogramas 9 y 10 muestran que la vocal corta / $\Lambda$ / en la palabra / bıd / 'bird' pájaro dura 0,179 milisegundos, mientras que la vocal larga / vo / en la palabra / buod / 'board' tabla tarda 0,266 milisegundos. 
Espectrograma 9

La vocal / ^ / en palabra / bud / 'bird' pájaro

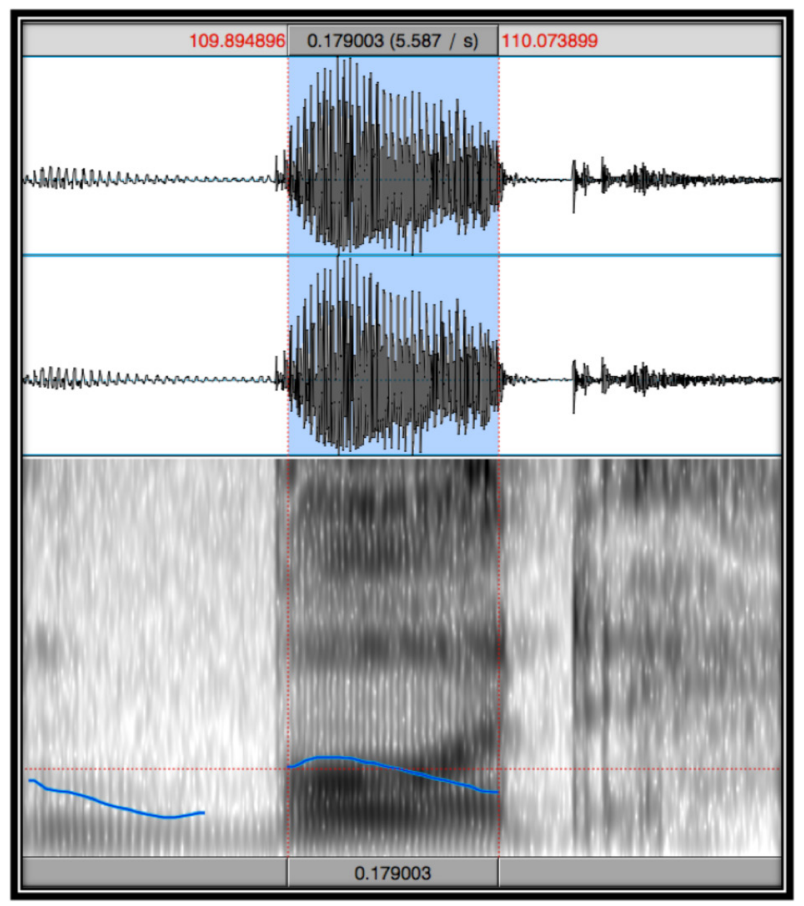

Espectrograma 10

Las vocales / 0 o / en palabra / bood / 'board' tabla

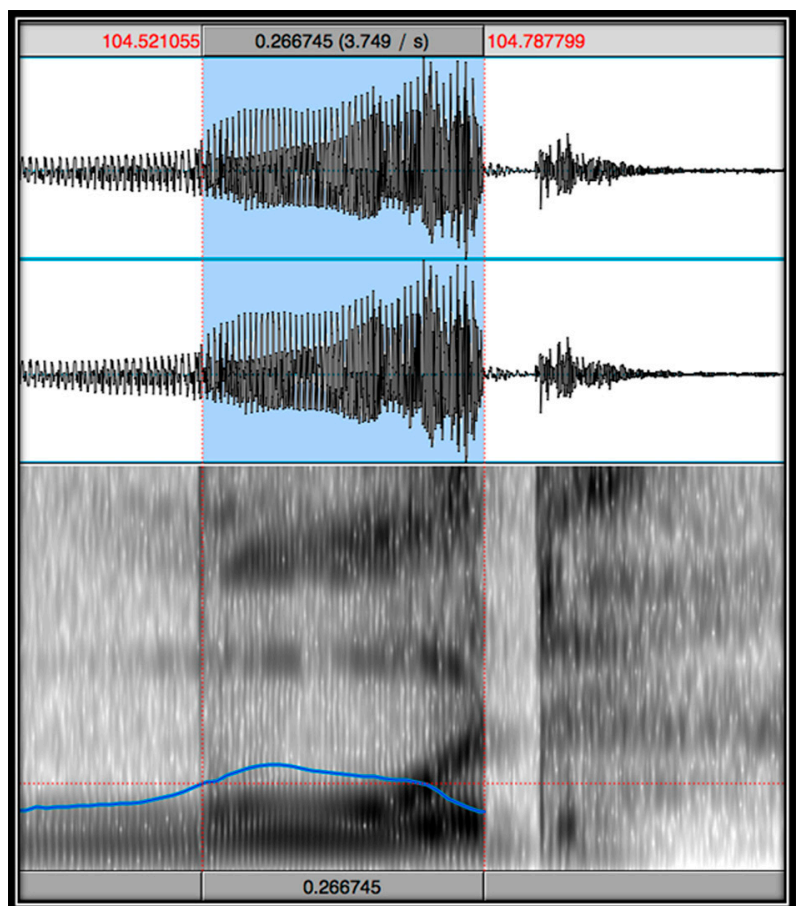


El espectrograma 10 muestra, además, que efectivamente esta vocal larga está compuesta de un diptongo. La primera parte del diptongo (correspondiente a la primera mora) dura 0,173 milisegundos. Esta consiste en una vocal alta, posterior, floja [ $v$ ]. En esta vocal, el F1 alcanza una frecuencia de $576 \mathrm{~Hz}$. Este valor ocupa un lugar intermedio respecto del promedio de la frecuencia del F1 establecido para los casos de la vocal alta tensa posterior / u: /, que es $510 \mathrm{~Hz}$, y del promedio de la frecuencia de la F1 para los casos de la vocal alta floja / v /, que es de $655 \mathrm{~Hz}$.

La segunda parte del diptongo (correspondiente a la segunda mora) tarda apenas 0,093 milisegundos. Esto equivale aproximadamente a una tercera parte de la totalidad del diptongo. Esta se compone de una vocal media, posterior, tensa [ o ]. La vocal presenta el F1 con una frecuencia de $526 \mathrm{~Hz}$, que es bastante menor que el F1 que muestra la vocal media, posterior,

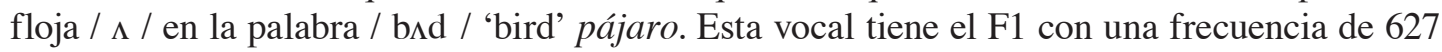
Hz. Como se ha dicho (cf. nota 3), el segmento / $\Lambda$ / puede describirse articulatoriamente como una vocal posterior centralizada, media, floja [ 0 ].

Por ello, también se debe señalar que el F2 de la vocal [ o ] posee una frecuencia de $1248 \mathrm{~Hz}$, la cual es menor que la frecuencia del F2 presentada en la vocal [ 0 ] de $1615 \mathrm{~Hz}$. Esto demuestra que, aunque se trata siempre de una vocal posterior, el segmento [ $\ddot{0}$ ] es más centralizado.

En el caso de estos diptongos, no hay rastro de las transiciones aproximantes o semicosonánticas ni antes ni después de la vocal [ o ] aducidas por Herzfeld (2002).

Respecto de las vocales medias anteriores, los espectrogramas 11 y 12 muestran que la vocal corta / e / en la palabra / belı / 'belly' estómago dura 0,125 milisegundos, mientras que la vocal larga / ie / en la palabra / tiela / 'tailor' sastre tarda 0,213.

Espectrograma 11

La vocal / e / en palabra / belı / 'belly' estómago

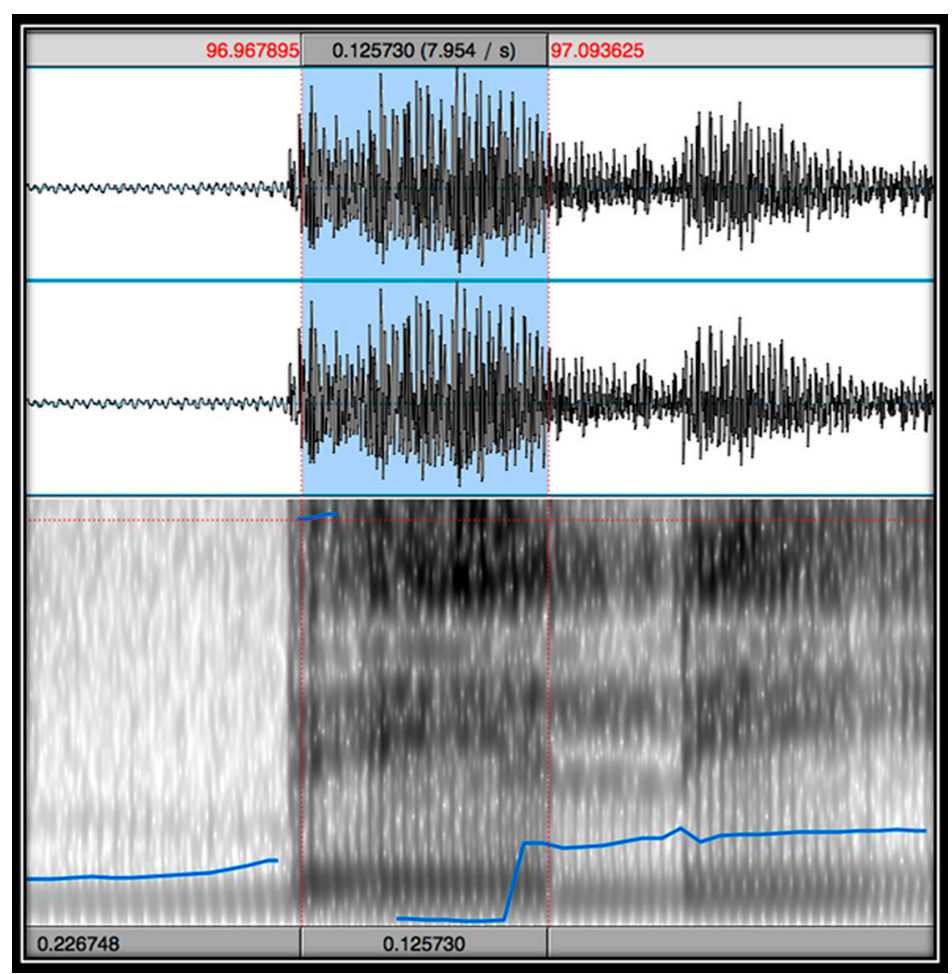


Espectrograma 12

Las vocales / ie / en palabra / tiela / 'tailor' sastre

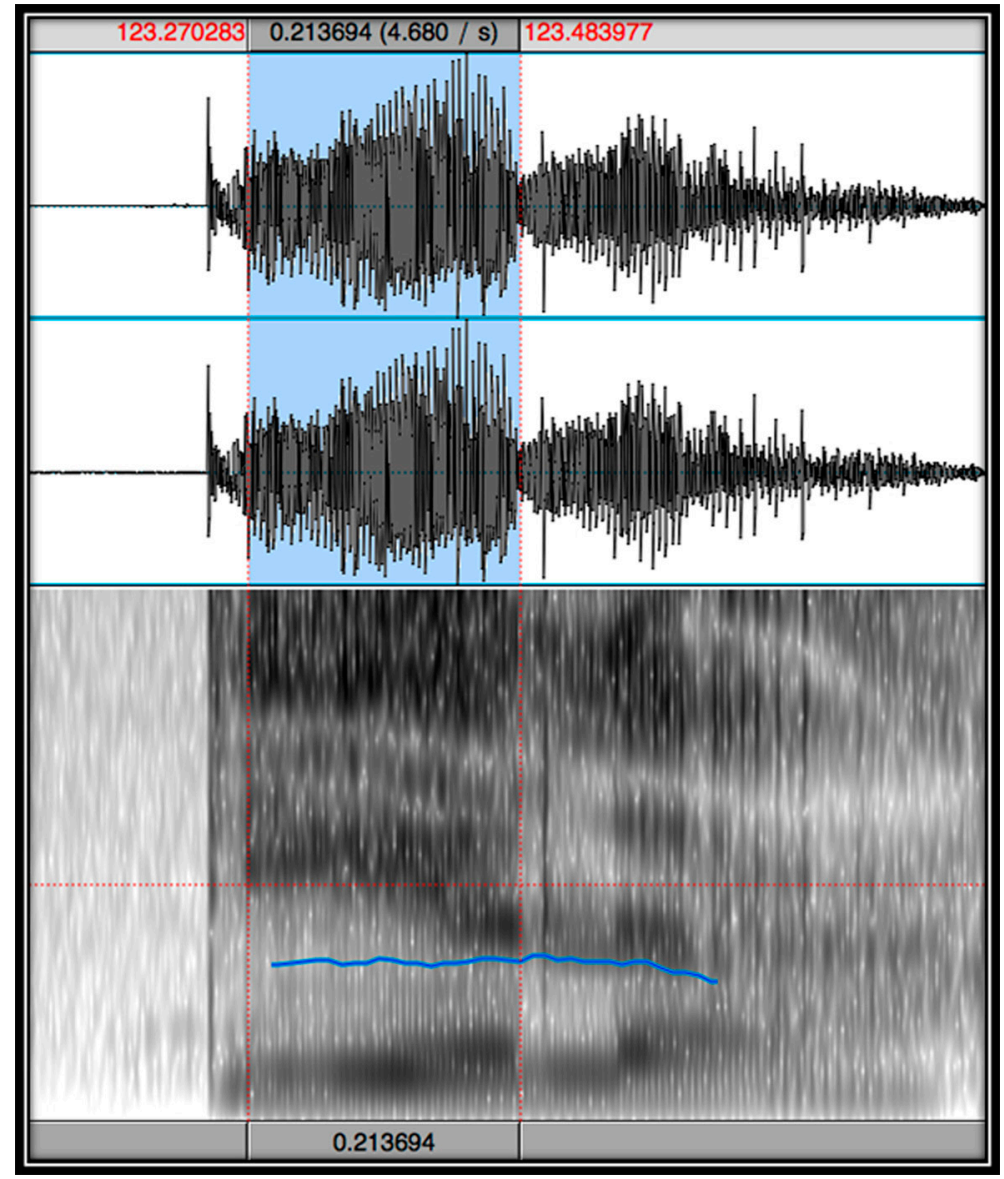

Además, el espectrograma 12 muestra que la vocal larga anterior corresponde a un diptongo. La primera parte del diptongo (o primera mora) consiste en una vocal alta, anterior, floja [ I ], cuyo F1 tiene una frecuencia de $422 \mathrm{~Hz}$. Este valor también ocupa un lugar intermedio respecto de los promedios de las frecuencias del F1 de las vocales altas anteriores. El promedio de la frecuencia de F1 para la vocal / i: / es de $369 \mathrm{~Hz}$, mientras que el de la frecuencia de F2 para la vocal / I / es de $408 \mathrm{~Hz}$.

La segunda parte del diptongo (o segunda mora) presenta una vocal media, anterior, tensa [ e ]. La frecuencia del F1 es de $534 \mathrm{~Hz}$ y la del F2 es de 2161. En contraste con el caso de las vocales medias posteriores, estos valores son muy parecidos a los que presenta la vocal anterior media / e / en esta lengua, que en promedio son los siguientes: la frecuencia del F1 es de $525 \mathrm{~Hz}$ y la del F2 es de 2199.

La primera parte del diptongo dura 0,141 y la segunda tarda 0,072 del total de 0,213 milisegundos. Al igual que se ha dicho para el caso de las vocales medias posteriores, en este caso la segunda parte del diptongo constituye casi exactamente un tercio de este. Por último, hay que señalar que el espectrograma 12 tampoco muestra las transiciones aproximantes o semiconsonánticas mencionadas por Herzfeld (2002). 


\section{Conclusiones}

En general, las vocales largas duran aproximadamente el doble de las vocales cortas, especialmente cuando son pronunciadas en palabras aisladas. Esta diferencia en la longitud persiste en las palabras que contienen dos vocales iguales, como se muestra en el espectrograma 13, que corresponde a la palabra / fa:da / 'father' padre.

Espectrograma 13

La palabra / fa:da / 'father' padre

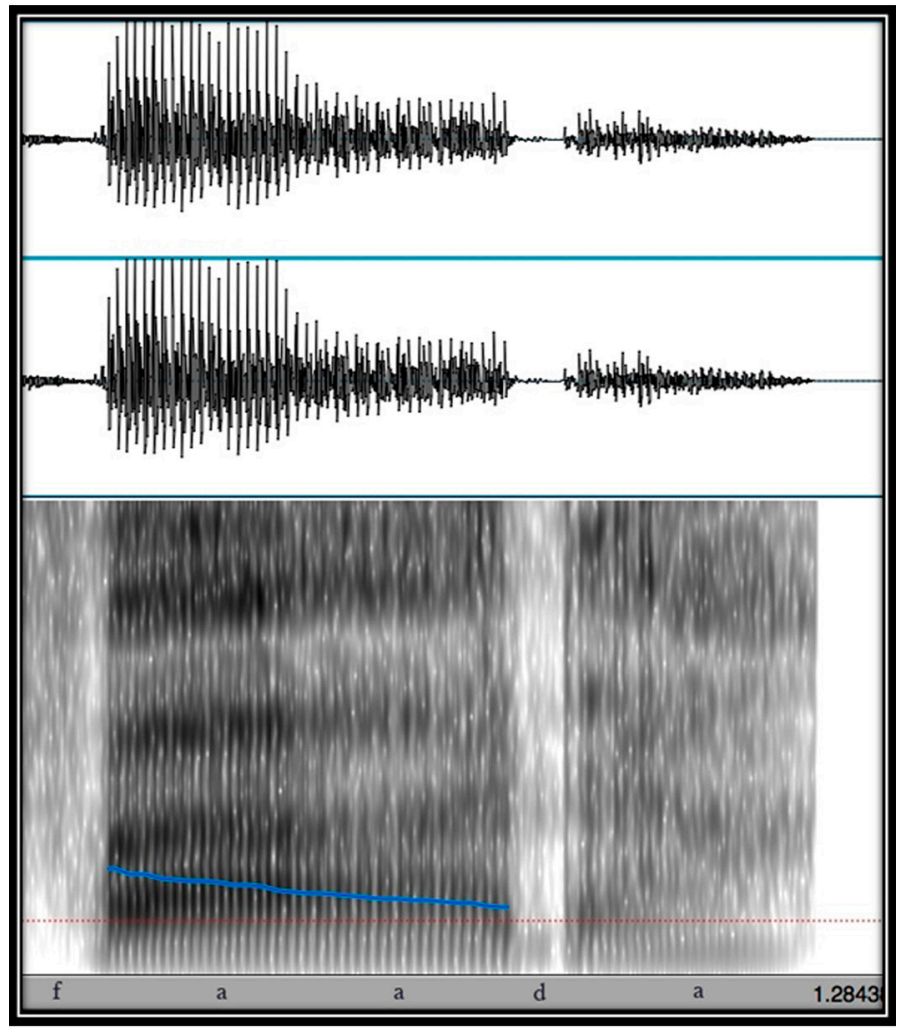

En la palabra / fa:da / 'father' padre, la vocal larga dura 0,259 milisegundos y la vocal corta 0,138 . En este caso, también la diferencia en duración de la vocal larga respecto de la corta es de casi el doble. En frases, la diferencia entre vocales largas y cortas es de solamente un tercio en promedio.

Por otro lado, los espectrogramas han mostrado que una vocal larga está compuesta de dos moras. En las vocales bajas y altas, la longitud de las moras tiende a ser muy semejante. Sin embargo, en las vocales medias (o diptongos), la primera mora ocupa dos terceras partes del segmento y la segunda mora solo una tercera parte.

El análisis de los espectrogramas permite determinar con certeza que las vocales largas no presentan transiciones aproximantes o semiconsonánticas de ninguna naturaleza, vgr. [ j, w, h ], ni siquiera en el caso de los diptongos descendentes / Ie, vo /, como se afirma en Herzfeld (2002).

El análisis de los formantes F1 y F2 permite ofrecer escalas de altura y posición de la lengua para las vocales en este idioma. El primer formante $(\mathrm{F} 1)$ corresponde a la apertura 
del tracto vocal, que proporciona información sobre la altura vocálica. Las vocales más bajas presentan una frecuencia mayor del F1 y las más altas una menor.

Así, se puede establecer una escala de altura para las vocales en esta lengua:

Frecuencias del F1 de las vocales en el inglés criollo de Limón

$$
\begin{aligned}
& \text { Vocales +altas } \\
& / \mathrm{i} \text { / }=369 \mathrm{~Hz} \\
& / \mathrm{i} /=408 \mathrm{~Hz} \\
& / \mathrm{ie} /=445 \mathrm{~Hz} \\
& / \mathrm{e} /=525 \mathrm{~Hz} \\
& / \mathrm{u} /=510 \mathrm{~Hz} \\
& / \mathrm{vo} /=559 \mathrm{~Hz} \\
& / \mathrm{\Lambda} /=627 \mathrm{~Hz} \\
& / \mathrm{v} /=655 \mathrm{~Hz} \\
& / \mathrm{a}:=975 \mathrm{~Hz} \\
& / \mathrm{a} /=976 \mathrm{~Hz} \\
& \text { Vocales +bajas }
\end{aligned}
$$

Como se ha dicho antes, quizá lo más llamativo de esta escala es que la vocal floja / u / se realiza fonéticamente de manera muy baja en la cavidad oral.

El segundo formante (F2) determina la posición de lengua en la cavidad oral. Las frecuencias más altas indican que la vocal se produce en una zona anterior de la cavidad oral y las más bajas en una posterior.

Con base en estos valores, se puede establecer una escala de posición de las vocales en la cavidad oral en esta lengua.

Frecuencias del F2 de las vocales en el inglés criollo de Limón

$$
\begin{gathered}
\text { Vocales +anteriores } \\
/ \mathrm{i} /=2485 \mathrm{~Hz} \\
/ \mathrm{Ie} /=2395 \mathrm{~Hz} \\
/ \mathrm{e} /=2223 \mathrm{~Hz} \\
/ \mathrm{I} /=2200 \mathrm{~Hz} \\
/ \mathrm{a} /=1888 \mathrm{~Hz} \\
/ \mathrm{u} /=1882 \mathrm{~Hz} \\
/ \mathrm{a} /=1814 \mathrm{~Hz} \\
/ \mathrm{\Lambda} /=1615 \mathrm{~Hz} \\
/ \mathrm{u} /=1504 \mathrm{~Hz} \\
/ \text { vo } /=1157 \mathrm{~Hz} \\
\text { Vocales +posteriores }
\end{gathered}
$$

En el caso de la escala del F2, lo más llamativo es que la frecuencia de la vocal alta larga / u: / es muy alta, y cae dentro del rango de las frecuencias de las vocales centrales en esta lengua. 


\section{Notas}

1. La interpretación fonológica por medio de rasgos distintivos es del autor de este artículo. Igualmente, el autor ha normalizado las transcripciones fonológicas utilizando los símbolos del Alfabeto Fonético Internacional.

2. Esta autora utiliza el símbolo " $y$ ” para representar la consonante aproximante palatal sonora [ j ]. Por otro lado, representa fonéticamente los segmentos fonológicos / ijh / como [ ije ] o [ je ].

3. El equivalente de la vocal / $\Lambda$ / de Portilla (1996) es / o / en Portilla (1993). Esto se debe a que, en realidad, este segmento se realiza fonéticamente como una vocal posterior centralizada media floja redondeada [ $\mathrm{o}]$.

4. Se ha omitido el señalamiento de los tonos altos en estas transcripciones.

5. Por ejemplo, Wolfe (1970: 2) señala que [ a ] y [ a: ] may also occur in all positions in free variation.

6. Resulta sorprendente que Wolfe no mencione el diptongo ascendente que aparece en la palabra inglesa cow, como sí lo hacen todos los demás autores.

7. Se trata de Marcia Reid Chambers, una hablante nativa de inglés criollo, de 55 años, quien ha sido una reputada consultora lingüística por muchos años.

8. Quiero expresar un especial agradecimiento a la Dra. Sandra Schwab por los conocimientos y motivación que me brindó en el Taller de Fonética Experimental llevado a cabo en agosto de 2009 en la Universidad de Costa Rica. Sin ellos, no hubiera podido realizar esta investigación.

9. Más comúnmente esta palabra se utiliza como término peyorativo para referirse a una persona afeminada. Se trata de un calco semántico tomado del español de Costa Rica (vid. Quesada Pacheco 2007: 290).

\section{Bibliografía}

Herzfeld, Anita. 1978. "Tense and aspect in Limon Creole: A socio-linguistic view towards a creole continuum". Tesis doctoral: Universidad de Kansas.

2002. Mekaytelyue. La lengua criolla. San José: Editorial de la Universidad de Costa Rica.

Portilla, Mario. 1993. "Fonemas segmentales en el criollo inglés de Limón". Revista de Filología y Lingüística de la Universidad de Costa Rica. 19(2): 89-97.

1995. "Tono en el criollo inglés de Costa Rica". Revista de Filología y Lingüística de la Universidad de Costa Rica. 21(1): 135-139.

1996. "Una ortografía para el criollo inglés de Costa Rica". Revista de Filología y Lingüística de la Universidad de Costa Rica. 22(2): 87-103. 
Quesada Pacheco, Miguel Ángel. 2007. Nuevo diccionario de costarriqueñismos. Cartago: Editorial Tecnológica de Costa Rica.

Wolfe, Terry. 1970. "An exploratory study of the morfology (sic) and syntax of the English of the Province of Limon, Costa Rica”. Tesis de licenciatura: Universidad de Costa Rica.

Wright, Fernando. 1974. "Limon Creole: A syntactic analysis". Tesis de licenciatura: Universidad de Costa Rica.

1975. "Un análisis sintáctico del habla criolla de Limón". Revista de Filología y Lingüística de la Universidad de Costa Rica. 1(2): 149-168. 
